Research Article

\title{
Discrete Dynamic Modeling and Change Trend Analysis of Regional Economy Based on Big Data
}

\author{
Yang Chen (iD) and Yu Yu (iD \\ Department of Management Engineering, Xuzhou University of Technology, Xuzhou 221000, China \\ Correspondence should be addressed to Yang Chen; chenyang@xzit.edu.cn
}

Received 10 September 2021; Revised 22 October 2021; Accepted 26 October 2021; Published 9 December 2021

Academic Editor: Gengxin Sun

Copyright (c) 2021 Yang Chen and Yu Yu. This is an open access article distributed under the Creative Commons Attribution License, which permits unrestricted use, distribution, and reproduction in any medium, provided the original work is properly cited.

\begin{abstract}
The driving force of high-quality development of regional economy is inseparable from the support of technology. With the support of big data, we need to solve this problem in order to solve the difficulty of large-scale experimental testing and accurately reflect the feasibility growth of data sample changes. This paper proposes a discrete dynamic modeling technology based on big data background to analyze the development and change of regional economy. The reliability AMSAA model is usually used for dynamic discrete modeling. It can be combined with the change data provided by big data to form a dynamic modeling method for reliability growth evaluation. Then, the Bayesian regression method is used to predict the change parameters of the model, and the spatial econometric method is used to analyze the regional economic change. The results show that compared with the traditional methods, the discrete dynamic modeling method is more accurate and can effectively solve the problem of reliable growth under the condition of big data. After introducing the spatial effect measurement model, it can also reflect the main factors of the growth and change of regional economic real output value. In addition to the development of high and new technology, terrain factors, investment, and government support have also had different effects. Therefore, according to the above results, it is proved that the discrete dynamic modeling technology can accurately obtain the experimental data and provide reliable technical support for dynamic data processing.
\end{abstract}

\section{Introduction}

With the continuous development of regional economy, the statistical work related to the current situation of regional economic development and influencing factors has become a key task [1]. In the context of big data, the statistics of regional economic influencing factors and changes have gradually shifted from complex work to the process of resource utilization using Internet technology $[2,3]$. In order to study the influencing factors and trends of regional economic changes, we use the network information function to process the data, and then we process the data to realize reuse for the objects of regional economic problems. Among them, the transformation, collection, and storage of data are the work contents of big data statistics. We need to actively study and discuss how to use the idea of big data and high data processing and apply it to regional economic problems [4].
At present, the imbalance of China's regional economic development has been one of the key concerns of researchers. Since the implementation of reform to optimize and adjust the supply side and drive innovation, all regions are actively developing high-tech industries and carrying out industrial transformation $[5,6]$. However, due to geographical factors and development conditions, the western region still has a certain gap from the eastern development region. This regional economic imbalance is also reflected in the per capita economic index GDP. Up to 2020, the provinces in the central and western regions have gradually changed from not meeting the standard to meeting the standard. Before 2020, Sichuan Province was still lower than the national index standard [7]. In terms of scientific and technological innovation in various provinces, the proportion of economic expenditure and the number of patented inventions are lower than the national average [8]. We study 
the time series changes of technical efficiency in various provinces and regions and the matrix distribution of regional efficiency values. The results show that the technical efficiency values in the eastern region are high, and the remaining regions are generally low $[9,10]$. Among them, there is still a positive correlation between technical efficiency and regional economic development. In the face of the above situation, the research on the impact of technical efficiency on regional economic change is proposed, and the discrete dynamic system modeling technology supported by big data is adopted [11]. In the use of small-cost data, largescale experimental research is realized, so as to solve the error problem caused by insufficient data capacity. This complex system reliability growth evaluation model should consider the rationality and operability in the growth process [12].

This paper is mainly divided into three parts. The first part introduces the current situation of technical efficiency in the influencing factors of high-quality development of regional economy under the background of big data and the application of prediction modeling methods adopted by various countries in the exploration of influencing factors of regional economic development. The second part first uses the dynamic modeling method of complex system reliability growth to explore the impact of technical efficiency on regional economic development and then uses spatial econometrics based on big data to analyze the factors of regional economic change. The third part analyzes the research results of complex system discrete dynamic modeling technology in the impact of technical efficiency on regional economic development, analyzes the research results of regional economic change factors under spatial econometric research, and analyzes the causes of regional economic development and change.

\section{Related Works}

From the active economic development in China's developed regions, we can know that the indicators of the main regional economies include investment in fixed assets, regional bank deposits in the whole year, regional bank loans in the whole year, foreign trade export amount, high-tech efficiency development projects, and so on [13]. With the development of big data, traditional statistical methods have been more common. The work efficiency of traditional methods is also relatively low, the consumption of human resources is huge, and with a high error rate, they are not applicable to the research on regional economic development and change at this stage $[14,15]$. At present, some studies believe that for the performance testing stage of China's regional economic development indicators in recent years, only static data can show the current situation and the gap from the standard target [16]. It is far from reflecting the regional economic development efficiency and structural changes supported by big data, let alone explaining the gap factors between the central and western regions and the eastern region. Therefore, we will explore the development status of dynamic modeling in various countries [17].

Some scholars have applied discrete dynamic modeling technology to human-computer interaction, including the interactive system of dynamic content, that is, the hybrid enhancement function of virtual reality [18]. Then, it developed into the monitoring system and gradually became the main research goal. This dynamic modeling technology greatly enriched people's cognition and understanding of the dynamic user interface. However, the hidden problem is that the interaction of underlying mechanisms and the complexity of problem tasks limit the development of dynamic modeling model.

In the field of geological survey, researchers use dynamic interpretation modeling technology for geological model construction framework $[19,20]$. This technology can respond to the construction process of geological layer modeling and fuse geological and geophysical data. The three-dimensional dynamic model is used to establish and display the contents of geological survey, which greatly facilitates the progress of port road survey, radar detection, and so on.

Some staff mainly established a dynamic system for the organization and formation of knowledge personnel in hightech enterprises and specifically studied the impact and prediction of recruitment system on the success rate of recruiters [21]. In the process of discrete dynamic modeling, the impact of job transfer and resignation on the data is also considered. The nonlinear discrete dynamic model established by them can provide recruitment strategy for the elastic control of employee system and change the stability of the system.

In previous studies on construction process in China, dynamic parametric 3D modeling technology was also used for construction management in the face of influencing factors such as complex environment, huge data, and difficult coordination $[22,23]$. It is convenient to understand the definition of engineering parameters and realize the detection of construction process, so as to reflect the realtime construction state, engineering measurement, and risk assessment [24].

According to the above research on discrete dynamic modeling technology, we know from the factors of regional economic development that the innovation driving force and technological efficiency progress have obvious advantages in the change of economic efficiency in the developed regions of eastern China [25]. However, effective evaluation methods are still needed to support which indicators are effective for regional economic changes. Therefore, this paper adopts discrete dynamic modeling technology to obtain and process dynamic economic data. Finally, the main factors of regional economic change are analyzed by means of spatial measurement [26]. 


\section{Research on Regional Economic Development Factors and Change Trend Based on Discrete Dynamic Modeling Technology under the Background of Big Data}

3.1. Research on Influencing Factors of Regional Economy Based on Complex System Reliability Growth Dynamic Modeling Technology. With the development of big data, a large amount of data is needed to support the calculation information in dealing with some relevant regional economic change factors and trends. Therefore, a large amount of data guidance is needed in the analysis of economic indicators in different regions, which is conducive to improving the quality of regional economic survey. At present, China's per capita GDP in the eastern region is better than that in other regions. With the increase of years, the gap between regional economies is becoming more and more obvious, and the momentum of rapid development is becoming stronger and stronger. There is also an obvious gap in the total amount of national economic development. The proportion of GDP in each region is shown in Figure 1.

As can be seen from Figure 1, the proportion of the central region has increased compared with previous years, and the trend of improvement in the western region is greater. Although the proportion of the eastern region used to be very large, it has declined and contracted, and the upward trend is gradually poor. The provinces in Northeast China have an obvious downward curve. In this regard, we need to study what factors cause the unbalanced impact on regional economic development. In the big data survey, we found that there is a linear relationship between technical efficiency and regional economic development. The AMSAA model is a widely used reliability growth model, and its interval estimation coefficient of MTBF is urgently needed in engineering. This paper gives some results, but it is far from showing the overall needs of engineering practice. The AMSAA model and Bayesian method are used to study the experiment. The purpose of introducing Bayesian method is to be applicable to any capacity data sample, and the reliability dynamic modeling can objectively evaluate the parameter distribution for analysis. Such methods can effectively save the cost of research. The Bayesian network model is shown in Figure 2.

It can be seen from Figure 2 that the probabilistic Bayesian network can actively deal with the simultaneous relationship representation and data analysis in multiple directions. It uses a directed acyclic graph as a network structure for data processing and calculation, which can solve the problems such as the dynamic change of regional economic survey data.

The AMSAA model is divided into two ways: quantity specified truncation and time specified truncation. In this paper, we set up an independent control group by censoring the specified time in the research process. We need to consider the factor of scale benefit in the research on the change

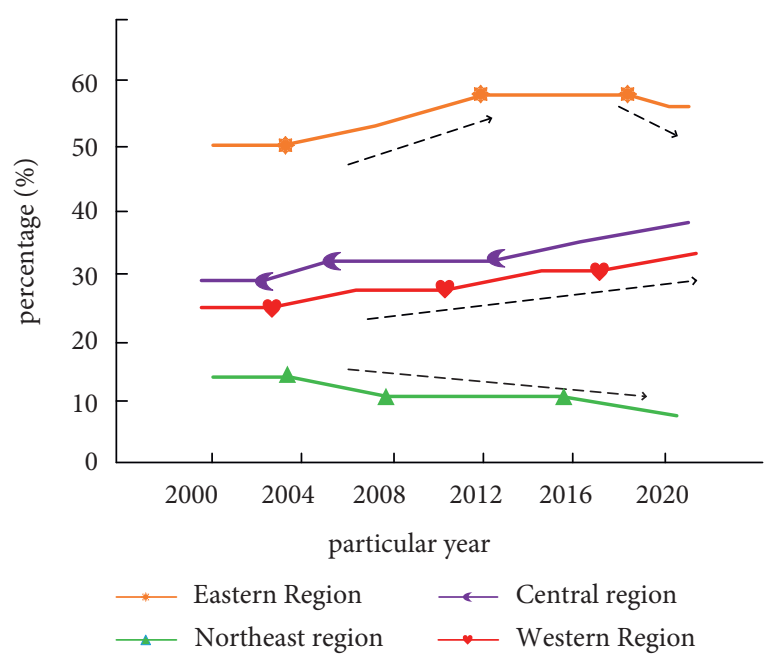

Figure 1: Proportion of GDP in each region.

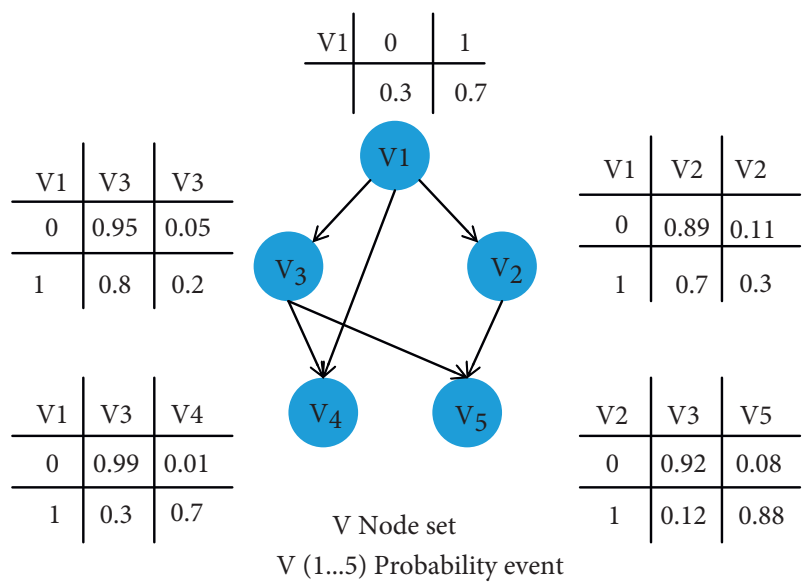

FIgURE 2: Bayesian network model diagram.

of regional economic income. We increase the expected value under the given conditions as a way to improve efficiency and carry out pretreatment. First, it is necessary to check whether the reliability still has growth changes. If there are no special changes, it cannot be estimated. If there is a significant upward change, we need to check whether the data comply with the discrete reliability model, which is calculated by using the classical prediction formula as follows:

$$
C_{M}^{2}=\frac{1}{12 M}+\sum_{i=1}^{M}\left[\left(\frac{t_{i}}{T}\right)^{b}-\frac{2 i-1}{2 M}\right]^{2},
$$

where $M$ is the number of operations in the research stage, $T$ is the test duration, and $t_{i}$ is the cumulative duration of stage fault problems. If it conforms to the reliability model, go to the step of parameter Bayesian analysis. The general analysis and calculation are very complex, and the numerical integration method is used for accumulation. We need to give the stage parameter distribution, and the expression formula is as follows: 


$$
m\left(t_{i 1, \ldots, t_{\text {ini }}}\right)=\int_{\substack{a i>0 \\ \text { bi }\left[b_{1}, b_{2}\right]}} f\left(t_{i 1}, \ldots t_{i n i} \mid a_{i}, b_{i}\right) \pi\left(a_{i}, b_{i}\right) d a_{i} d b_{i}
$$

After calculation, the following two formulas can be derived:

$$
\begin{aligned}
m\left(t_{i 1, \ldots,}, t_{i n i}\right)= & \frac{r\left(a_{i}+n_{i}\right) \beta_{i}^{a i}}{r\left(a_{i}\right)\left(b_{2}-b_{1}\right) \prod_{i=1}^{n i} t_{i} n_{i}} \\
& \cdot \int_{b_{1}}^{b_{2}} b_{i}^{n i} \prod_{i=1}^{n i} t_{i n i}^{b_{i}}\left(\tau_{i}^{b_{i}}+\beta_{i}\right)^{-\left(a_{i}+n_{i}\right)} d b_{i} .
\end{aligned}
$$

Set the variables $\beta_{i}$ and $a_{i}$ to 0 to obtain the output variable to obtain the estimated value. The approximate method used in the above preprocessing integral calculation process is limited, so it is difficult to determine the distribution parameters of the AMSAA model. Based on the above situation, we need to convert the data to obtain the estimated value of effective parameters and reflect the growth of reliability model through failure rate. In the process of effective dynamic evaluation, how to predict the failure variable $\lambda_{i}^{\wedge}$ is usually calculated by historical data. From the classical calculation formula of historical stage:

$$
\lambda_{i}^{\wedge}=\frac{{ }^{n} D_{i}}{{ }^{\tau} D_{i}},
$$

where ${ }^{n} D_{i}$ and ${ }^{\tau} D_{i}$ represent the historical information of each stage, respectively. The basic theory of dynamic modeling is to establish dynamic parameters from historical data and mechanisms at different levels and finally form a prediction model. This prediction model is used to determine the numerical reliability distribution at the current stage, and then Bayesian algorithm is used for numerical prediction based on big data. Discrete linear computing is widely used. Compared with the traditional model statistics, its excellent computational characteristics are more suitable for the dynamic modeling process in this paper. In dynamic modeling, multiple coincident values need to be linearly combined, and the influence degree of factors is analyzed according to the characteristic quantity, that is, the development trend of failure rate and reliability. The change of function diagram, failure curve, and reliability curve is shown in Figure 3.

As can be seen from Figure 3, when the data volume is stable and sufficient and the function group distance is getting closer and closer, an approximately smooth curve at the top can be obtained. There is an inverse correlation between reliability and failure rate. For the AMSAA model, the expression of failure degree is

$$
\lambda_{i}=a(1-m) T_{i}^{-m}
$$

where $a$ and $m$ are distribution parameters. It is defined that the former is greater than 0 and the latter is between 0 and 1 . Set the total closing time of all stages, and the following model formula can be obtained according to the value:

$$
\widehat{y}_{i}=H_{i} \mu+\varepsilon_{i}, \quad i=1 \ldots N
$$

where

$$
\begin{aligned}
H_{i} & =\left(1, x_{i}\right), \\
\mu & =\left(\beta_{0}, \beta_{1}\right) .
\end{aligned}
$$

The above formula is in matrix form, $\mu$ is the regression coefficient variable to be estimated, and the offset estimation of the model can be calculated by substituting the historical data information into the model as the control value. In order to solve the problem of dynamic information change, we use memory elimination to replace the input data according to the stage data iteration. According to the above formula, the relationship structure between the gain/loss efficiency linear model and the calculation object is calculated. After the reliability is proved by the failure rate, the dynamic Bayes estimation needs to be carried out, and the gamma distribution, that is, the continuous probability function, is used to calculate the model variables. First, calculate the mean and variance formulas as follows:

$$
\begin{gathered}
E\left[\lambda_{i} \mid \mu\right]=\frac{\beta_{i}}{\alpha_{i}} \\
D\left[\lambda_{i} \mid \mu\right]=\frac{\beta_{i}}{\alpha_{i}^{2}}
\end{gathered}
$$

The calculation formula after numerical conversion is

$$
\begin{gathered}
\alpha_{i}=\frac{H_{i} \widehat{\mu} L, S}{\sigma_{\Pi}^{2}}, \\
\beta_{i}=\left(\frac{H_{i} \widehat{\mu} L, S}{\prod_{\sigma}}\right)^{2} .
\end{gathered}
$$

After the above calculation, the distribution nature of the impact of the sample change of technical efficiency on the regional economy can be predicted. For example, after the conditional samples are obtained after the random stage test, after the model conversion, the effective experimental data still follow gamma distribution. The formula is as follows:

$$
\prod\left(\lambda_{i} \mid\left(N_{D i}, T_{D i}\right)=g\left(\lambda_{i} ; a_{i}+T_{D i}, \beta_{i}+N_{D i}\right) .\right.
$$




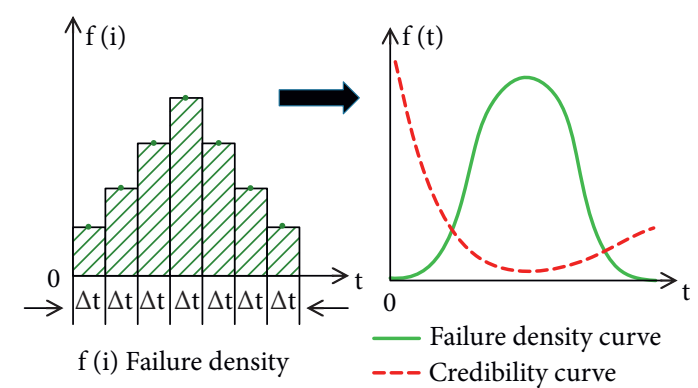

Figure 3: Variation diagram of function, failure curve, and reliability curve.

The failure rate of the AMSAA model is an observable value. Because the distribution property can be used for data iteration during the equivalent value conversion operation, there is no need to set up dynamic equation in dynamic modeling. We select the number of industrial technological activities in each region from the perspective of scientific and technological innovation investment and study the impact of technical efficiency on regional economic changes according to the proportion of industrial expenditure and technological transformation investment in each region. The data come from the scientific and technological statistical report of previous years and relevant regional statistical data as a supplement. In the face of the above big data calculation, AMSAA discrete dynamic modeling can effectively process data information and obtain reliability relationship. The calculation results confirm that technical efficiency has a positive correlation impact on regional economic changes.

\subsection{Research on Spatial Measurement Technology of Regional} Economic Change Dynamic System Based on Big Data. After studying the dynamic modeling of the impact of technical efficiency on regional economy, this paper makes an empirical study on the current situation and influencing factors of China's regional economic development based on the theory of regional economics and the theory of big data and spatial econometric model. Firstly, the global spatial variable correlation verification is used, and the definition formula is

$$
\text { Moran's } I=\frac{\sum_{i=1}^{n} \sum_{j=1}^{n} w_{i}\left(Y_{i}-\bar{Y}\right)\left(Y_{j}-\bar{Y}\right)}{s^{2} \sum_{i=1}^{n} \sum_{j=1}^{n} w_{i j}} .
$$

The definition of $s^{2}$ in the relation is used to represent the change of numerical value in a certain region. The observed value is $Y_{i}$ and $n$ is the total number of areas. Use the spatial matrix calculation method to take the quantity value uniformly. If the effect is less than 0 , it indicates a negative correlation, and when it is close to a negative number, it becomes stronger and stronger. Equal to 0 means uncorrelation, greater than 0 means positive correlation, and close to 1 means stronger and stronger.

In local spatial correlation analysis, the definition formula is

$$
I_{i}=\frac{\left(x_{i}-\bar{x}\right)}{s^{2}}=\sum_{j} w_{i j}\left(x_{j}-\bar{x}\right) .
$$

Among them, the positive $I_{i}$ variable indicates that the spatial unit is similar to the adjacent numerical relationship attribute. The negative value of $I_{i}$ indicates that it is not similar, that is, the height is scattered. The specific comparison is shown in Figure 4. follows:

The definition formula of spatial weight matrix is as

$$
\begin{aligned}
& W=\left[\begin{array}{l}
w_{11} w_{12} \ldots w_{1 n} \\
w_{21} w_{22} \ldots w_{2 n} \\
w_{n 1} w_{n 2} \ldots w_{n n}
\end{array}\right], \\
& w_{i j}=\left\{\begin{array}{l}
\frac{1}{i \wedge j}, \\
0 .
\end{array}\right.
\end{aligned}
$$

It indicates that the two regional variables $i$ and $j$ are adjacent to each other. If the regions are adjacent to each other in the spatial measurement during the calculation, then $w_{i j}$ is equal to 1 , indicating that there is an obvious spatial relationship between the regions. If the regions are not adjacent to each other, it is expressed as 0 , which means that they have no spatial relationship and are independent of each other. In the study of regional economic development factors, we mainly select the per capita GDP of Guangdong Province of China as the data support for the global spatial correlation survey. Global spatial data Moran were compared with mean $P$ and the statistical gap $Z$-value, respectively. The empirical results after big data spatial measurement test are shown in Figure 5. It is to determine whether there is a correlation between spatial entities within a certain range, such as residential buildings, and whether they are gathered together or scattered everywhere. Moran index values are distributed in $[-1,1] ;[0,1]$ indicates that there is a positive correlation between geographical entities, and $[-1,0]$ indicates that there is a negative correlation, while 0 has no correlation.

It can be seen from Figure 5 that the Moran values of per capita GDP in Guangdong Province are positive and have passed the significant level test. The statistical gap between the data is also within the controllable range to ensure the reliability of the results. It also proves that there is an obvious spatial correlation between regional economies. That is, the distribution of China's economic growth among different regions is not random but is jointly affected by the neighboring regions. According to the above examples, we conducted a spatial econometric study on the regional distribution throughout the country and compared the Moran value change trends in the central region, the eastern region, the western region, and the northeast region, respectively, as shown in Figure 6. 


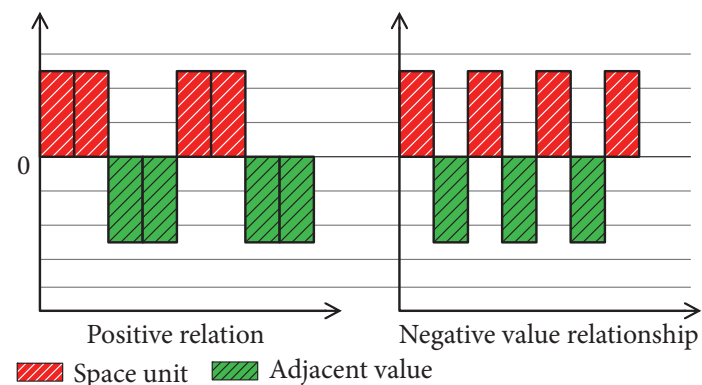

FIGURE 4: Illustration of positive value relationship and negative value relationship.

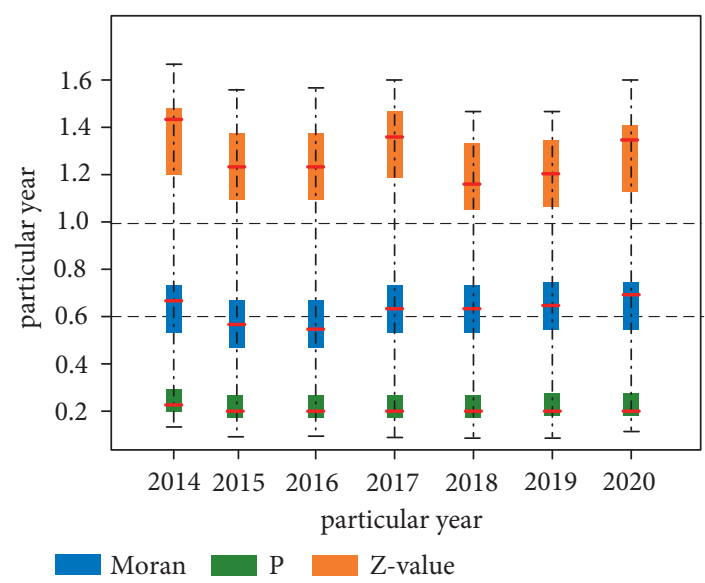

FIGURE 5: Empirical diagram of global spatial coefficient of GDP.

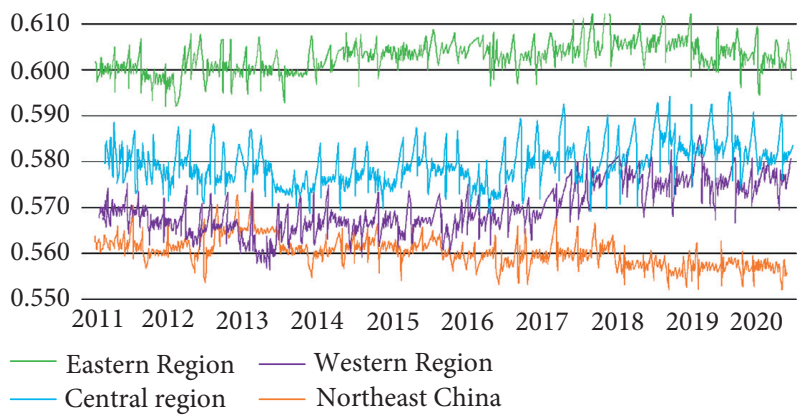

Figure 6: Change trend of Moran value in China.

As can be seen from Figure 6, the region with the best regional economic development is the eastern region, which gradually increases with the increase of years, but there is an obvious downward trend in the last few years. The early development of Northeast China is good, and there is an obvious downward trend with the increase of years. This unbalanced regional economic situation shows that there is spatial correlation in the national urban economy. The factors causing regional economic changes are also derived from the development of high-tech industries to the development of adjacent cities. Finally, the regional radar map is generated according to the change of per capita GDP of spatial econometric statistics to reflect the change rate of the overall economic development index. The influencing factors are investment amount, high-tech development, terrain advantage, import and export economy, financial allocation, and per capita GDP, as shown in Figure 7.

It can be seen from Figure 7 that the rapid development of the eastern region is related to factors such as topographic advantages, import and export trade, high-tech development, and so on. The development of high and new technology in the central region drives the growth of other economic factors. The economic growth rate in the western and northeast regions is slow or even declines due to the lack of geographical environment and the slow development of high and new technology. Therefore, this paper puts forward some suggestions on the current data research results: the development of adjacent cities can be used to drive regional economic development, and government departments can improve the introduction of technology development in remote areas and drive economic integration through capital investment to reduce the per capita burden.

\section{Analysis of Research Results on Regional Economic Development Factors and Change Trend Based on Discrete Dynamic Modeling Technology under the Background of Big Data}

4.1. Analysis of Research Results of Influencing Factors of Regional Economy Based on Complex System Reliability Growth Dynamic Modeling Technology. This paper selects China's regional provinces and cities as the basic test data samples and classifies them according to the traditional regional division. In the calculation, it can be seen that the reliability has increased significantly. According to the discrete dynamic modeling, the technical efficiency (TE) and the decomposed pure technical efficiency (PTE) are calculated, and the change diagram of technical efficiency and pure technical efficiency over the years in the corresponding region is made, as shown in Figure 8.

As can be seen from Figure 8, with the time series change characteristics of the region, we can know that the average value of the whole region is rising in the process of annual growth. It also proves that there is a gradual upward trend in China's overall technical efficiency. The overall curve of the western region changes obviously, and the central region remains basically stable. The overall technical value of the eastern region is higher than that of the whole region. The northeast region as a whole has declined but will be basically stable. Through the above statistics, we can conclude that the regional technology drive is obvious and can affect the changes of the overall regional economy. The use of dynamic modeling technology can intuitively obtain the accurate data of calculation and solve the problem of evaluation in the face of a small amount of small sample data in complex systems. Compared with the traditional discrete modeling mechanism, reliability dynamic modeling can improve the accuracy of research data. 


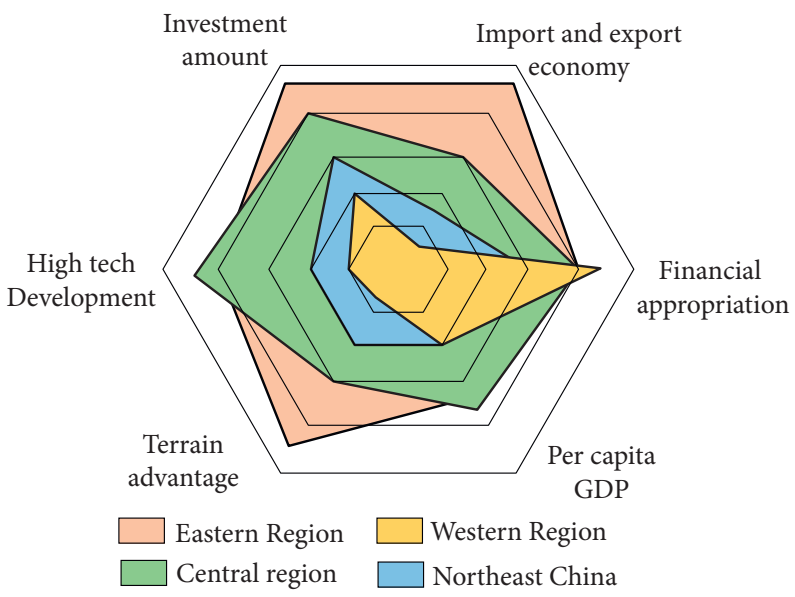

FIGURE 7: Influence chart of reflecting factors on the change rate of regional economic development index in China.
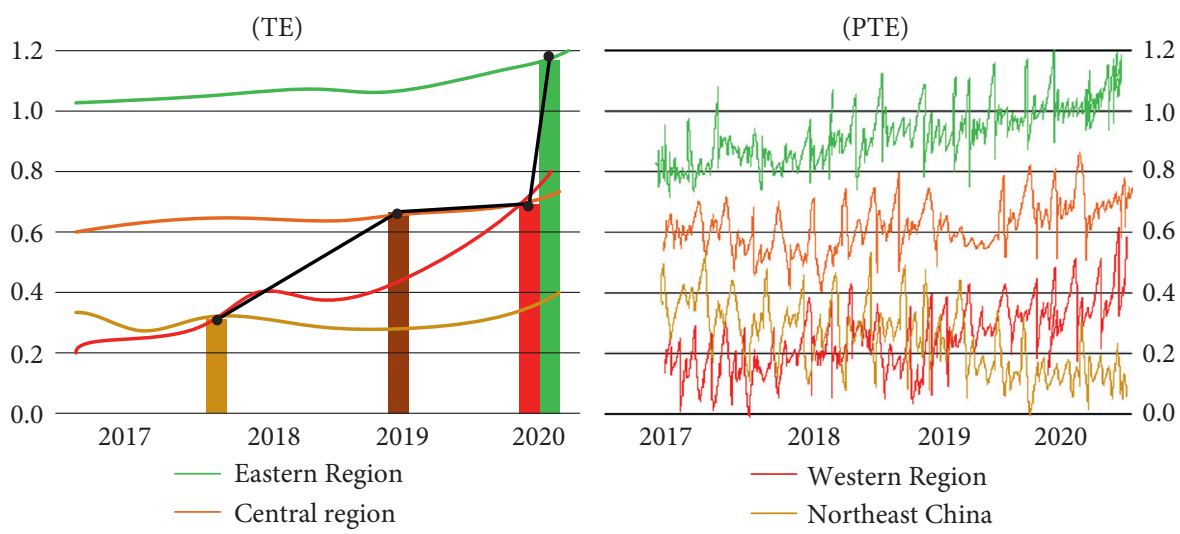

Figure 8: Comparative change diagram of TE and PTE in each region.

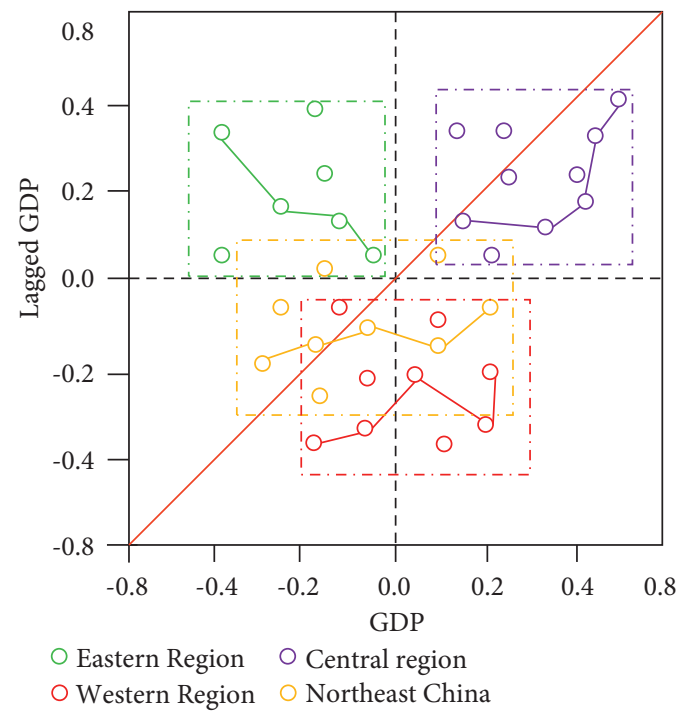

FIGURE 9: Scattered spatial map of GDP aggregation in each region.

4.2. Analysis of Research Results of Spatial Measurement Technology of Regional Economic Change Dynamic System Based on Big Data. We use big data as experimental data to study regional economic changes. Through the spatial econometric analysis of the above dynamic system, we roughly conclude that regional economic changes are related 


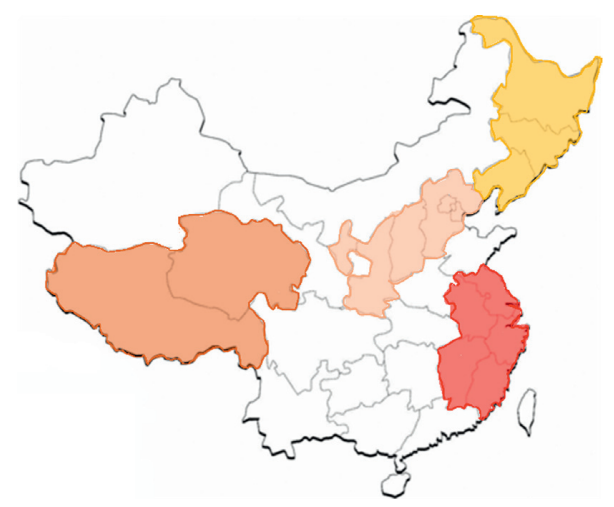

Low-Low

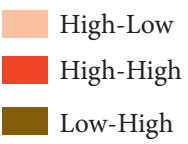

FIGURE 10: Influence diagram of significant coefficient of regional economic GDP.

to the development of adjacent cities. The global spatial correlation test found that there was an obvious spatial correlation in per capita GDP, that is, the growth of regional economy was not random but had a common impact. This paper mainly takes the provincial units as the research object and makes a scattered spatial prominence analysis on the regional GDP agglomeration, as shown in Figure 9.

As can be seen from Figure 9, the scatter diagram formed by GDP index and stagnation index in various regions is mainly shown as spatial positive correlation and negative correlation. Each region has obvious spatial aggregation effect, the eastern region has high aggregation state, and the northeast region is widely distributed in each quadrant. We construct a map model divided by the impact of national and regional economic GDP, as shown in Figure 10. The national and regional economic GDP is divided into four parts: lowlow, high-low, high-high, and low-high.

Therefore, using dynamic discrete space econometric analysis of regional economic change trend has obtained effective results. The main changes of regional economy come from many aspects. In addition to technical efficiency and topographic factors, the development space of adjacent cities also has a positive or negative impact on regional economy.

\section{Conclusion}

With the development of the big data era, we gradually apply it in various fields, such as military research, news media, medical images, economic research, and so on. At present, the factors of regional economic change have always been our focus. This paper uses discrete dynamic modeling technology and dynamic spatial measurement technology under the background of big data to explore and analyze the factors of regional economic change. Compared with the traditional discrete linear mathematical model, the dynamic modeling technology can deal with the error caused by data changes and improve the numerical accuracy in the process of analyzing regional economy. According to the analysis of the research results of discrete dynamic data graph, technical efficiency is an important factor in regional economic development. In this paper, dynamic data modeling and Bayesian network structure are used to analyze the impact on technical efficiency. The results show that the dynamic modeling technology can accurately solve the evaluation problem of reliability growth of complex dynamic data and clarify the influencing factors of regional economic change. In addition, this paper makes an overall analysis of the global change of regional economy according to the dynamic spatial econometric algorithm. The results show that the adjacent space of regional provinces and cities has positive and negative influencing factors. In addition to the development of high and new technology, terrain factors, investment amount, government support, and other links have also had different effects. Therefore, according to the above results, practice has proved that discrete dynamic modeling technology can accurately obtain experimental data and provide reliable technical support for dynamic data processing. The conversion can be realized in multisample data and small-sample data to achieve the same experimental purpose. However, after dynamic modeling, the relevant data are not simulated in this paper. Therefore, the computer simulation value still lacks verification, which needs to be given in the future research.

\section{Data Availability}

The data used to support the findings of this study are included within the article.

\section{Conflicts of Interest}

The authors declare that they have no conflicts of interest. 


\section{Acknowledgments}

This study was supported by the Social Science Fund Project (Xuzhou) (Research on innovation factor flow and collaborative innovation of urban agglomeration in Huaihai Economic Zone) (no. 20XSZ-073).

\section{References}

[1] J. Guo and Y. Tian, "Research on high-quality economic development of the Yangtze river economic belt based on five development concepts," Statistics and management, vol. 36, no. 9, pp. 53-59, 2021.

[2] X. Chen and Z. Miao, "Growth power of digital economy and spatial distribution law of regional income," Journal of Geography, vol. 76, no. 8, pp. 1882-1894, 2021.

[3] C. Lin, "Research on agricultural economic management measures under the background of new rural construction," Agricultural Development and Equipment, vol. 8, pp. 60-61, 2021.

[4] W. Zhao, "Analysis on the impact of highway transportation, logistics and transportation on regional economic development," Investment and Cooperation, vol. 8, pp. 90-91, 2021.

[5] J. Huang, "Correlation analysis between regional economic growth and logistics industry agglomeration under the background of big data," China Logistics and Procurement, vol. 24, p. 48, 2020.

[6] Y. Lai and Q. Sun, "Spatial econometric analysis of regional economy in Guangdong Province based on big data," China Economic and Trade Guide (Middle), vol. 12, pp. 70-72, 2020.

[7] K. Xiao and H. Qian, "Application of big data in regional economic statistics," Science, Technology and Economy Guide, vol. 29, no. 2, pp. 8-9, 2021.

[8] D. Wu, "Cluster analysis of China's regional economic coordination based on big data," Global Outlook on Science, Technology and Economy, vol. 35, no. 1, pp. 55-59, 2020.

[9] W. Zhang, X. Ye, L. Dong, J. Fu, and M. Wu, "Network correlation, spatial spillover effect and China's regional economic growth-a study based on Tencent's location big data," Geoscience, vol. 39, no. 9, pp. 1371-1377, 2019.

[10] W. Yi, "Research on using big data to improve regional economic forecasting and decision-making ability-Taking Nanchong real estate market as an example," Business news, vol. 20, pp. 11-13, 2019.

[11] H. Xu, L. He, and H. He, "Application of big data in regional economy for industrial structure optimization," China Statistics, vol. 10, pp. 10-12, 2018.

[12] L. Lin, "Research on the value and Realization of Pan Organization collaborative application in developing regional economy under the environment of big data and cloud computing," Computer products and circulation, vol. 12, pp. 152-153, 2018.

[13] Y. Ning, Management and Decision-Making Big Data Platform for Regional Economic Monitoring and Market Subject Service, Guangzhou Cuckoo Information Technology Co., Ltd., Guangzhou, China, 2017.

[14] B. Tang, H. Fu, and C. Tang, "Design of model dynamic modeling system in special complex test device," Computer Measurement and Control, vol. 28, no. 9, pp. 229-232, 2020.

[15] S. Duan, "Dynamic path of academic English chunk development research: complex system modeling," Journal of Foreign Languages, vol. 1, pp. 87-91, 2019.

[16] Y. Zhang, T. Chai, and J. Yang, "Alternating identification algorithm and application for a class of nonlinear discrete- time dynamic systems," Journal of automation, vol. 43, no. 1, pp. 101-113, 2017.

[17] D. Dai, Z. Cai, and B. Zhang, "Discrete dynamic system modeling and elastic control of knowledge workers in hightech enterprises," Journal of China University of metrology, vol. 32, no. 1, pp. 109-117, 2021.

[18] X. Yu, Z. Hou, M. M. Polycarpou, and L. Duan, "Data-driven iterative learning control for nonlinear discrete-time MIMO systems," IEEE Transactions on Neural Networks and Learning Systems, vol. 32, no. 3, pp. 1136-1148, 2020.

[19] A. Zhirabok, "The problem of invariance in nonlinear discrete-time dynamic systems," Symmetry, vol. 12, no. 8, 2020.

[20] L. Zhang, "Discrete event dynamic system and its application in network simulation," Wireless interconnection technology, vol. 15, no. 22, pp. 36-38, 2018.

[21] L. Peng and J. Cheng, "3D modeling technology of coal seam in mining face and its application in intelligent mining," Coal Mine Safety, vol. 52, no. 8, pp. 156-161, 2021.

[22] T. Wang, Y. Wang, S. Niu, and L. Yao, "Dynamic modeling and prediction of soil moisture based on real-time moisture content data," China Agrometeorology, vol. 42, no. 1, pp. 13-23, 2021.

[23] L. Liu, F. Wu, G. Zhang, and Y. Bai, "Research and application of tunnel construction monitoring method based on dynamic parametric 3D modeling technology," Modern tunnel technology, vol. 57, no. S1, pp. 859-863, 2020.

[24] S. Cai, Y. Jing, G. Liu et al., "Dynamic modeling technology for weather sensitive load forecasting of smart grid based on Fisher information and online SVR," Chinese Journal of electrical engineering, vol. 40, no. 11, pp. 3441-3452, 2020.

[25] F. Nadir, "A discrete-event model of the train traffic on a linear metro line," Applied Mathematical Modelling, vol. 96, 2021.

[26] R. Davidrajuh, B. Skolud, and D. Krenczyk, "Performance evaluation of discrete event systems with GPenSIM," Computers, vol. 7, no. 1, 2018. 